\title{
The azimuthal distribution of thunderstorm events recorded by the GRAPES-3 experiment
}

\author{
B. Hariharan, ${ }^{a, *}$ S. Ahmad,${ }^{b}$ M. Chakraborty, ${ }^{a}$ A. Chandra, ${ }^{b}$ S.R. Dugad, ${ }^{a}$ \\ U.D. Goswami, ${ }^{c}$ S.K. Gupta, ${ }^{a}$ Y. Hayashi, ${ }^{d}$ P. Jagadeesan, ${ }^{a}$ A. Jain, ${ }^{a}$ P. Jain, ${ }^{e}$ \\ S. Kawakami, ${ }^{d}$ H. Kojima, ${ }^{f}$ S. Mahapatra, ${ }^{g}$ P.K. Mohanty,${ }^{a}$ R. Moharana, ${ }^{h}$ Y. Muraki ${ }^{i}$ \\ P.K. Nayak, ${ }^{a}$ T. Nonaka, ${ }^{j}$ A. Oshima, ${ }^{f}$ B.P. Pant, ${ }^{h}$ D. Pattanaik, ${ }^{a, g}$ G.S. Pradhan, ${ }^{k}$ \\ P.S. Rakshe, ${ }^{a}$ M. Rameez, ${ }^{a}$ K. Ramesh, ${ }^{a}$ L.V. Reddy, ${ }^{a}$ R. Sahoo, ${ }^{k}$ R. Scaria, ${ }^{k}$ \\ S. Shibata, ${ }^{f}$ J. Soni, ${ }^{e}$ K. Tanaka, ${ }^{l}$ F. Varsi ${ }^{e}$ and M. Zuberi ${ }^{a}$ \\ ${ }^{a}$ Tata Institute of Fundamental Research, Homi Bhabha Road, Mumbai 400005, India \\ ${ }^{b}$ Aligarh Muslim University, Aligarh 202002, India \\ ${ }^{c}$ Dibrugarh University, Dibrugarh 786004, India \\ ${ }^{d}$ Graduate School of Science, Osaka City University, Osaka 558-8585, Japan \\ ${ }^{e}$ Indian Institute of Technology Kanpur, Kanpur 208016, India \\ ${ }^{f}$ College of Engineering, Chubu University, Kasugai, Aichi 487-8501, Japan \\ ${ }^{g}$ Utkal University, Bhubaneshwar 751004, India \\ ${ }^{h}$ Indian Institute of Technology Jodhpur, Jodhpur 342037, India \\ ${ }^{i}$ Institute for Space-Earth Environmental Research, Nagoya University, Nagoya 464-8601, Japan \\ ${ }^{j}$ Institute for Cosmic Ray Research, Tokyo University, Kashiwa, Chiba 277-8582, Japan \\ ${ }^{k}$ Indian Institute of Technology Indore, Indore 453552, India \\ ${ }^{l}$ Graduate School of Information Sciences, Hiroshima City University, Hiroshima 731-3194, Japan \\ E-mail: 89hariharan@gmail.com
}

The GRAPES-3 experiment reported the measurement of $1.3 \mathrm{GV}$ potential across one of the massive thunderclouds recorded on 1 December 2014 by making use of the muon imaging technique. This measurement is ten times larger than the maximum potential reported previously by balloon and rocket sounding measurements, verifying the almost a century old prediction by C.T.R. Wilson. These measurements rely on the precise estimate of the change in the angular muon flux caused by the acceleration of muons during their passage through the charged layers of thunderstorms. The electric potential is estimated with the help of Monte Carlo simulations by using CORSIKA and other in-house tools. A study of the thunderstorms events recorded since April 2011 displays an asymmetry in their azimuthal distribution which can be understood to be caused by the ratio of $\mu^{+} / \mu^{-}$.

$37^{\text {th }}$ International Cosmic Ray Conference (ICRC 2021)

July 12th - 23rd, 2021

Online-Berlin, Germany

\footnotetext{
${ }^{*}$ Presenter
} 


\section{Introduction}

The record-breaking electric potential of 1.3 Giga-Volt $(\mathrm{GV})$ in one of the massive thunderclouds ever recorded by GRAPES-3 is the first direct experimental evidence proving C.T.R. Wilson's nearly a century old prediction [1]. Historically thundercloud voltages were measured using the best available yet inaccurate methods using balloon and rocket soundings. Unlike the previous methods of measurements, the recent study exploited muon imaging of thunderclouds. This technique is far superior compared to the conventional techniques where the problems of time skew and small sampling area are inevitable and limited by the technologies - thus leads to an underestimation by a factor of at least ten. Muons being relativistic they traverse the entire thundercloud instantaneously, therefore the muon imaging technique is a wise choice for tomography of the thundercloud. Along with the large sky coverage of GRAPES-3's field of view (FOV of $2.3 \mathrm{sr}$ ), it is possible to overcome the above mentioned problems and have an accurate estimation of thundercloud properties using muon flux variation. However, the interpretation of observed muon flux variation can be done through a detailed study on Monte Carlo simulations which itself rely on the hadronic interaction models built based on various theoretical assumptions and tuned with experimental results.

A detailed summary on angular distribution of 487 thunderstorm events recorded during April 2011 to December 2020 is presented here. Though the GRAPES-3 muon telescope (G3MT) is being operated for more than two decades, the electric field measurements required to study the thunderstorm effects are actually available since April 2011 with the installation of electric field mills (EFMs) around GRAPES-3. The observed distribution is found to have a significant asymmetry in East-West direction compared to North-South. Further investigations based on Monte Carlo simulations could explain the observed phenomenon found to be due to asymmetry in muon charge ratio $\left(\mu_{R}=\mu^{+} / \mu^{-}\right)$which is due to the effects of geomagnetic field. The Earth's magnetic field plays a crucial role in bending of particle trajectories in outside and inside the atmosphere for primary and secondary particles respectively.

\section{The GRAPES-3 muon telescope}

The G3MT is built using proportional counter (PRC) as a basic element. Each PRC is a $6 \mathrm{~m}$ long mild steel tube with a cross-section of $10 \times 10 \mathrm{~cm}^{2}$. The wall thickness of the PRC is $2.3 \mathrm{~mm}$. Both ends of the PRC are sealed and filled with P10 gas (a mixture of 90\% argon and 10\% methane). At the center a $100 \mu \mathrm{m}$ thick tungsten wire is laid that acts as anode where as the metal body being cathode. The PRC is supplied with a potential difference of about 3000 Volts DC that works as a detector based on gas ionization principle. The gas ionization produces an electron and a positively charged ion pairs, in short ion-pairs, gets multiplied by the large electric field around the anode and causes the avalanches. The methane additive in the P10 acts as a quenching gas to control avalanches. Height of the discharge pulse in the anode is proportional to the number of ion-pairs produced which is directly proportional to the energy deposited by the passing particle. The G3MT is organized into sixteen independent muon modules (3712 PRCs). Each module consists of four layers of PRCs. Each layer consists of a carpet of 58 PRCs tightly packed together. The layers are sandwiched by $15 \mathrm{~cm}$ thick concrete columns. Above the top most layer, $2 \mathrm{~m}$ thick concrete slabs are placed in the form of inverted pyramidal shape to provide an energy threshold of $\sec (\theta) \mathrm{GeV}$ up 

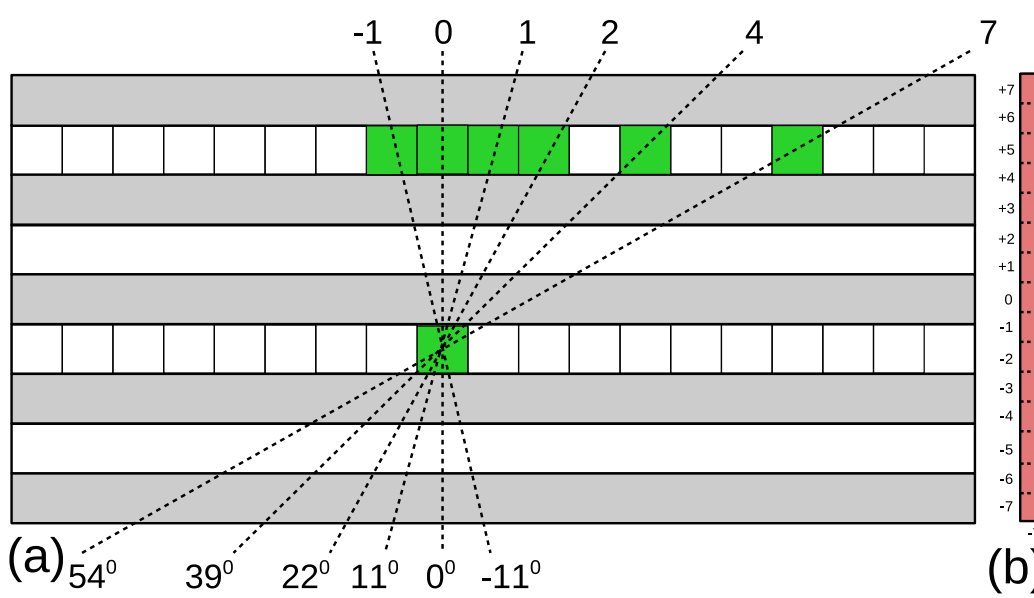

Figure 1: Representation of (a) muon direction reconstruction in a single projection using PRC hits and (b) grid of reconstructed $15 \times 15=225$ directions, the inner grid of $13 \times 13=169$ directions are shown by dashed lines, the number of actual usable directions for physics studies. The 169-direction configuration can be combined into $3 \times 3=9$ directions as shown by the solid lines. It is to be noted the unusual number of directions combined to yield the 9-direction configuration which is to provide similar solid angle coverage in each of the nine directions.

to $45^{\circ}$ (sky coverage of $2.3 \mathrm{sr}$ ). Such a massive absorber ensures the detection of $>1 \mathrm{GeV}$ muons with $\theta<45^{\circ}$ and filtering out the soft components. The alternate arrangement of layers allows to reconstruct the muons in 169 usable directions (Ref. Figure 1) for physics studies with a mean angular resolution of $4^{\circ}$. A detailed account of the G3MT can be found here [2]. The 169-direction configuration can be combined into $3 \times 3=9$ directions as shown by the solid lines in Figure $1 \mathrm{~b}$. It is to be noted that the unusual number of directions combined to yield the 9-direction configuration which is to provide similar solid angle coverage in each of the nine directions.

Each muon module records about 3000 muons every second. Everyday the G3MT records $4 \times 10^{9}$ angular muons with a small statistical error of $0.001 \%$. The muons are by-products of extensive air shower (EAS) phenomenon using atmosphere as a medium. So, any change in atmospheric properties can result in muon count. It is well understood that muons are highly sensitive to atmospheric pressure change and the same is corrected [3]. Also, the muon rate is successfully corrected for detector efficiency variation [4]. After all these systematic effects are taken care the high statistics data allow to measure any tiny change is muon flux caused by any physical phenomenon. Notable events such as measurement of more than billion volts in thunderclouds [1] and detection of short muon burst triggered by a geomagnetic storm [5,6] are evident that muons are good proxy to study near earth phenomenon.

\section{Monte Carlo simulations}

The observed change in muon intensity may be understood with the aid of Monte Carlo simulations of EAS development in the Earth's atmosphere. Majority of the cosmic rays consist of protons (i.e. $\sim 90 \%$ ). So, the simulations are carried out for a broad range of electric potential ranging from $-3 \mathrm{GV}$ to $3 \mathrm{GV}$ in steps of $0.1 \mathrm{GV}$. Uniform electric field is applied at an altitude of 
8-10 km above msl with electric field model implemented inside CORSIKA. The resultant muon intensity is derived in 169 directions using an in-house detector simulation tool. The simulated number of primaries are scaled in such a way that each direction of the G3MT should have at least $10^{6}$ muons for each potential step where as the background to have statistics ten times of the signal so that error can be brought down comparable to data. Figure 2 shows the simulated muon intensity variation as the function of applied electric potential in 9-direction configuration for the ease of visualisation. More details about the simulation can be found here [7]. The above said detailed simulations are carried out for combination of SIBYLL and FLUKA hadronic interaction models for high and low energies respectively. An independent study with various choices of other hadronic interaction models reveals that the estimated potential does not vary much among them [8].

It is important to have a brief description about the simulated muon intensity vs electric potential profiles in the context of the present work. The profiles of 9-direction configuration display distinct structures and help to estimate the electric potential in a specific direction. It can be explained with the muon charge asymmetry found in the nature. In the positive domain $(+\mathbf{V})$ the muon intensity reduces due to excess of $\mu^{+}$over $\mu^{-}$. Similarly, in the negative domain $(-\mathbf{V})$ the muon intensity increases as expected. However, beyond a certain potential the muon intensity decreases

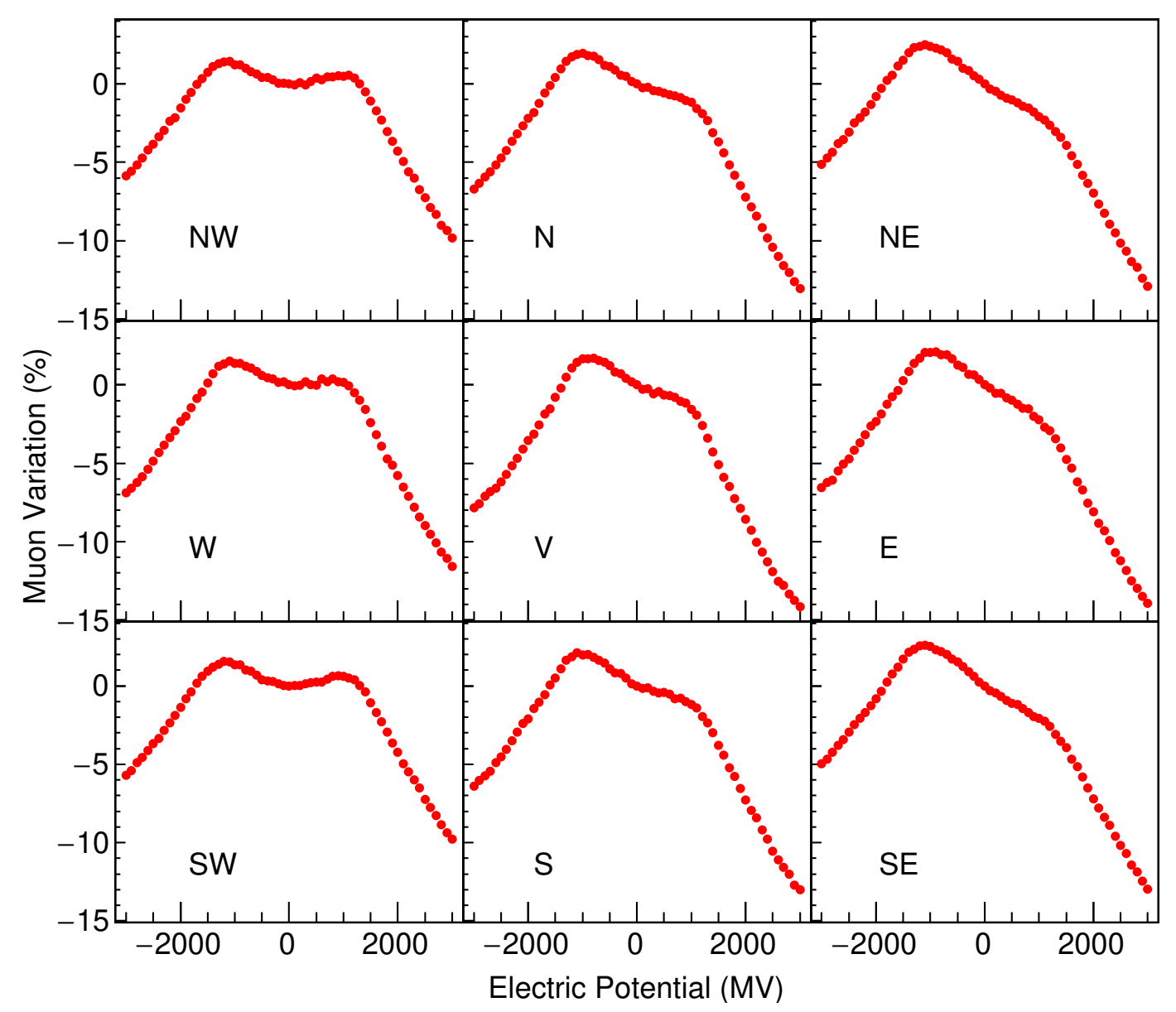

Figure 2: Monte Carlo simulation for GRAPES-3 FOV in 9-direction configuration. 
irrespective of the applied potential which can be attributed to the increased decay probability of muons beyond a certain energy (i.e. $\pm 1 \mathrm{GV}$ ). This can be also seen in the positive domain as well where as the change in slope implies increased decay of muons.

\section{Azimuthal distribution of thunderstorm events}

\begin{tabular}{c|rrc|c}
$\mathrm{NW}$ & \multicolumn{3}{|c|}{$\mathrm{N}$} & $\mathrm{NE}$ \\
\hline & 6.2 & 1.8 & 30.1 & \\
$\mathrm{~W}$ & 0.6 & 0.2 & 2.8 & $\mathrm{E}$ \\
& 7.0 & 2.8 & 48.6 & \\
\hline $\mathrm{SW}$ & & $\mathrm{S}$ & & $\mathrm{SE}$
\end{tabular}

Table 1: Percentage of thunderstorm events in GRAPES-3 FOV. Events in the vertical (V) direction is at the center of the table.

The G3MT records about 50 significant thunderstorm events every year. Though, the G3MT is in operation for more than two decades, the thunderstorm analysis is possible only since April 2011 after the installation of EFMs. The G3MT recorded 487 significant thunderstorm events during the period from April 2011 to December 2020. These events are analysed in the 9-directional configuration of the G3MT. The angular distribution of these events are displayed in Table 1. All North-South strips show a clear symmetry in the observed events compared to East-West strips.

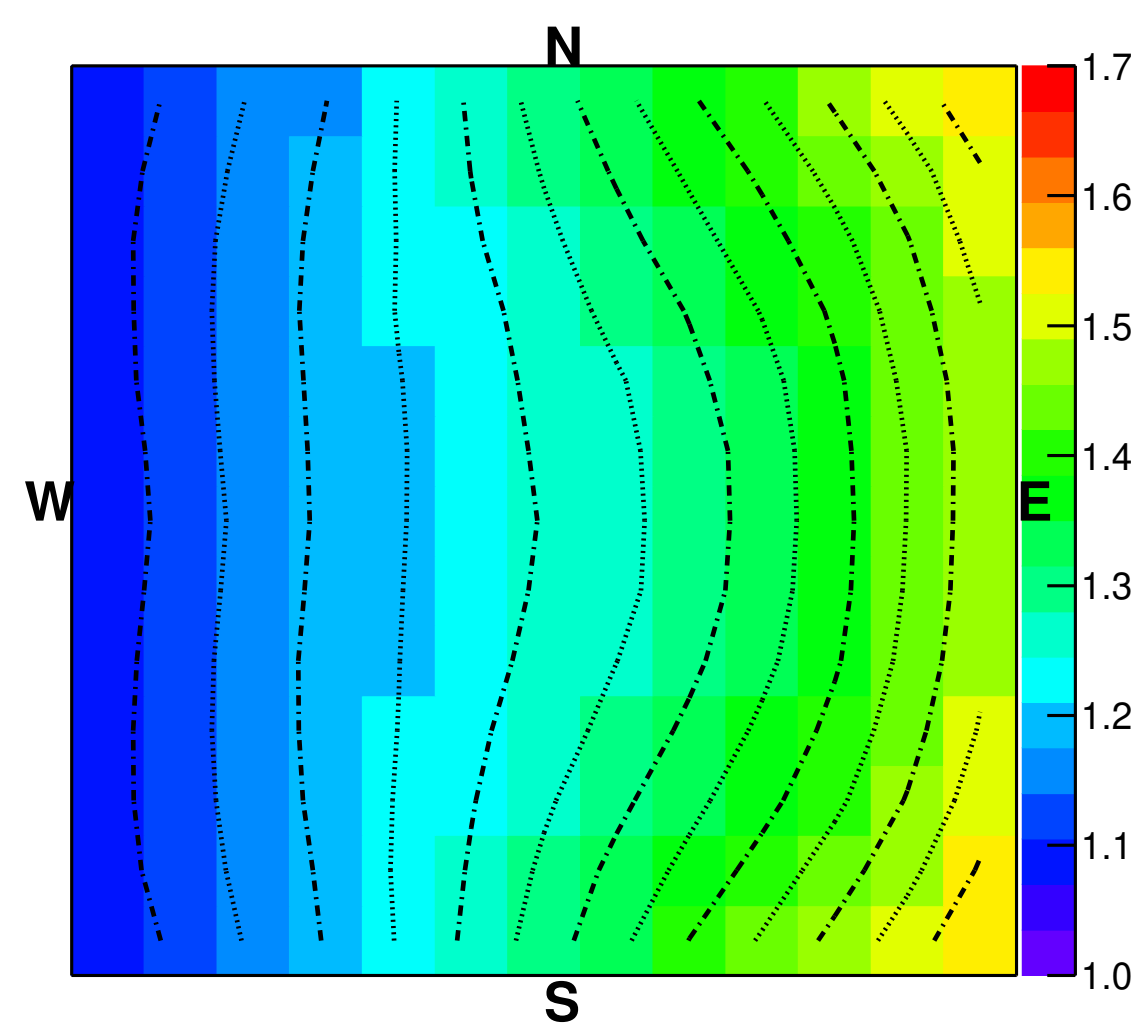

Figure 3: Distribution of $\mu_{R}$ for GRAPES-3 FOV in 169-direction configuration. 
Surprisingly, the percentage of events in the vertical and West directions are the smallest among the distribution. These symmetries are found to be caused by the $\mu_{R}$ due to the effects of geomagnetic field. The $\mu_{R}$ obtained from the Monte Carlo simulation is shown in Figure 3. It can be seen that the $\mu_{R}$ in the East direction is greater than one - hence, the $\mu^{+}$is decelerated for a positive potential and vice versa. Since, the G3MT measures the total muon flux $\left(\mu^{+}+\mu^{-}\right)$, considering the fact that the thundercloud potential is positive in most cases, majority of the events are found to be in the East (Figure 2). It can be also seen that the $\mu_{R}$ in the West and vertical directions is almost equal to one - hence, the changes in the total muon flux become insignificant.

\section{Conclusions}

A detailed study on angular distribution of 487 thunderstorm events recorded by the G3MT during a period of almost a decade revealed a clear East-West asymmetry. This is explained by examining the $\mu_{R}$ derived from the detailed Monte Carlo simulations. It is found that the $\mu_{R}$ showed a similar East-West asymmetry due to geomagnetic field. The $\mu_{R}$ plays a key role in impacting the measured muon flux due to the acceleration and deceleration of muons of different polarities. Since, the G3MT records the total muon flux, the observed net change is sensitive to the $\mu_{R}$ of the corresponding direction. This could be also the reason for the detection of smaller number of events in the West and vertical direction due to $\mu_{R}$ being almost equal to one.

\section{Acknowledgments}

We thank D.B. Arjunan, A.S. Bosco, V. Jeyakumar, S.Kingston, K. Manjunath, S. Murugapandian, S. Pandurangan, B. Rajesh, V. Santhoshkumar, M.S. Shareef, C. Shobana, and R. Sureshkumar for their efforts in maintaining the GRAPES-3 experiment. The TM-DAQ was developed with the support of FPGA based boards provided by ALICE collaboration.

\section{References}

[1] B. Hariharan et al., Phys. Rev. Lett. 122, 105101 (2019).

[2] Y. Hayashi et al., Nucl. Instrum. Methods A 545, 643 (2005).

[3] P.K. Mohanty et al., Astropart. Phys. 79, 23-30 (2016).

[4] P.K. Mohanty et al., Proceedings of Science PoS(ICRC2017)357.

[5] P.K. Mohanty et al., Phys. Rev. Lett. 117, 171101 (2016).

[6] P.K. Mohanty et al., Phys. Rev. D 97, 082001 (2018).

[7] B. Hariharan et al., Proceedings of Science PoS(ICRC2017)305.

[8] B. Hariharan et al., Proceedings of Science PoS(ICRC2019)186. 